\title{
Allergic rhinitis, bronchial asthma and other allergies in patients with Alzheimer's disease: unnoticed issue
}

\author{
Andrzej Bożek ${ }^{1}$, Piotr Bednarski², Jerzy Jarzab ${ }^{1}$ \\ ${ }^{1}$ Clinical Department of Internal Disease, Dermatology and Allergology in Zabrze, Medical University of Silesia in Katowice, Poland \\ Eleonora Reicher National Institute of Geriatrics, Rheumatology and Rehabilitation, Warsaw, Poland
}

Adv Dermatol Allergol 2016; XXXIII (5): 353-358

DOI: 10.5114/ada.2016.62842

\begin{abstract}
Introduction: Allergic diseases are becoming more prevalent in elderly patients. Allergic diseases have been observed in patients with Alzheimer's disease (AD). The prevalence of atopic bronchial asthma, allergic rhinitis and atopic dermatitis was analyzed in such elderly Polish population.

Aim: Analysis of the presence of allergic diseases in the patients with AD in Poland, including asthma, allergic rhinoconjunctivitis and atopic dermatitis.

Material and methods: The recruitment of subjects with AD was conducted at 6 sites representative of Polish rural and urban areas, and 1060 subjects with a mean age of $69.2 \pm 5.1$ years were screened. Medical examinations, an original questionnaire, skin prick testing for common aeroallergens and appropriate serum-specific lgE assays were performed.

Results: Probable atopy was diagnosed in 234 (22.1\%) analyzed patients, including 127 women (21.5\% of women) and 234 men (22.8\% of men). The average prevalence associated with age and sex in this population for bronchial asthma was $2.9 \%$, atopic dermatitis/eczema was $0.6 \%$, seasonal allergic rhinitis was $6.6 \%$, perennial allergic rhinitis was $11.1 \%$ and polymorphous atopic disease was $4.4 \%$. The most frequent positive results were recorded for the following allergens: mixed grass, Dermatophagoides pteronyssinus, Dermatophagoides farinae and Alternaria. Conclusions: One-fifth of diagnosed patients with $A D$ have allergic disease requiring treatment.
\end{abstract}

Key words: allergy, dementia, IgE, bronchial asthma.

\section{Introduction}

The problem of allergic diseases is increasing in most countries. Allergic rhinitis, bronchial asthma and atopic dermatitis are global health problems that affect the quality of life of all age groups [1, 2]. The increasing incidence of allergies and the ageing of populations have led to an increased incidence of atopic diseases in older patients and also in these with concomitant Alzheimer's disease (AD). Alzheimer's disease is a huge problem all over the world especially in elderly patients. This disease currently affects more than five million patients in the U.S. and will rise to 16 million by 2050. Globally, an estimated 35.6 million people have dementia (mainly AD), which is expected to reach 65.7 million in 2030 and 115.4 million in 2050 [1, 2]. In elderly patients, some chronic diseases can worsen cognitive function, depressive symptoms and functional status in AD. This pattern has been observed in many cases of heart failure, metabolic diseases such as diabetes, neoplastic diseases, chronic obstructive pulmonary disorder (COPD), bronchial asthma and others [3-5]. While many authors have analyzed the epidemiology, diagnostics and treatment of allergy, the occurrence and natural course of allergic disease in patients with $A D$ has not been explored much.

\section{Aim}

This study is an analysis of the presence of allergic diseases in the patients with AD in Poland, including asthma, allergic rhinoconjunctivitis and atopic dermatitis.

Address for correspondence: Andrzej Bożek MD, Clinical Department of Internal Disease, Dermatology and Allergology, Medical University of Silesia, 10 M. Skłodowskiej-Curie St, 41-800 Zabrze, Poland, phone: +48 3227131 65, e-mail: andrzejbozek@o2.pl Received: 17.07.2015, Accepted: 1.10.2015. 


\section{Material and methods}

The recruitment of subjects was conducted at 6 sites representative of central, southern and northern Poland. The selected areas included isolated rural centers (at 3 sites) and cities (at 3 centers). A sampling stratification was performed with a key criterion of age greater than 60 years. Within each database, comparable numbers of subjects were recruited from rural and urban centers. These groups were homogeneous regarding the age and sex ratio and were consistent with the demographic structure of the Polish population over 60 years of age in 2012. All patients with a positive history of AD were chosen from the basic group. It was 1060 patients. The study was performed in 2011-2012. The permission to publish the data was obtained from all of the centers. All of the subjects and guardians signed consent to participate in the study. The study was approved by the Bioethics Committee of Medical University School of Silesia in Katowice (Poland). Screening was performed by family doctors, geriatricians, internists or allergists, with the participation of trained nurses. The diagnosis of dementia of the Alzheimer's type was confirmed by criteria from the Diagnostic and Statistical Manual of Mental Disorder, Fourth Edition and the National Institute of Neurologic, Communicative Disorders and Stroke-AD and Related Disorder Associations [6, 7]. In all patients a diagnosis of AD was made using a combination of clinical criteria, neuropsychological testing and conventional computed tomogra- phy $(\mathrm{CT})$ and magnetic resonance imaging (MRI). Patients with a mild and moderate type of AD (II-IV degree on the Reisberg scale) were included into the study. Cognitive function was assessed on the basis of the Clock-Drawing Test and the Mini-Mental State Examination (MMSE) at the beginning of the study, after 6 months and after 1 year. The final score of MMSE was standardized according to the participant's age and years of education [8]. Medical histories of the patients including the history of allergic diseases were analyzed. Physical examinations and additional tests were performed. Alzheimer's disease was diagnosed by the cerebral CT. The patients with guardians completed a questionnaire about allergic diseases that was partly based on the short ECRHS II questionnaire as was shown in Table 1 [9]. Survey questions were included and concerned the type of atopic disease, its beginning and duration, and the symptoms of rhinitis, conjunctivitis, asthma, skin allergies and symptoms of hypersensitivity to the basic inhalation allergens. Next, a full medical examination was performed with a simplified otolaryngological examination (nasal rhinoscopy and a throat evaluation), dermatological evaluation and assessment of the eye. Skin prick testing with common inhalant allergens and assays of serum concentrations of total IgE and specific IgE were performed. The study was performed based on the Pharmacia CAP System FEIA (ThermoFisher, Sweden) immunoenzymatic method. The results of these assays were evaluated according to the manufacturer's instructions [10].

Table 1. Questionnaire about allergic diseases

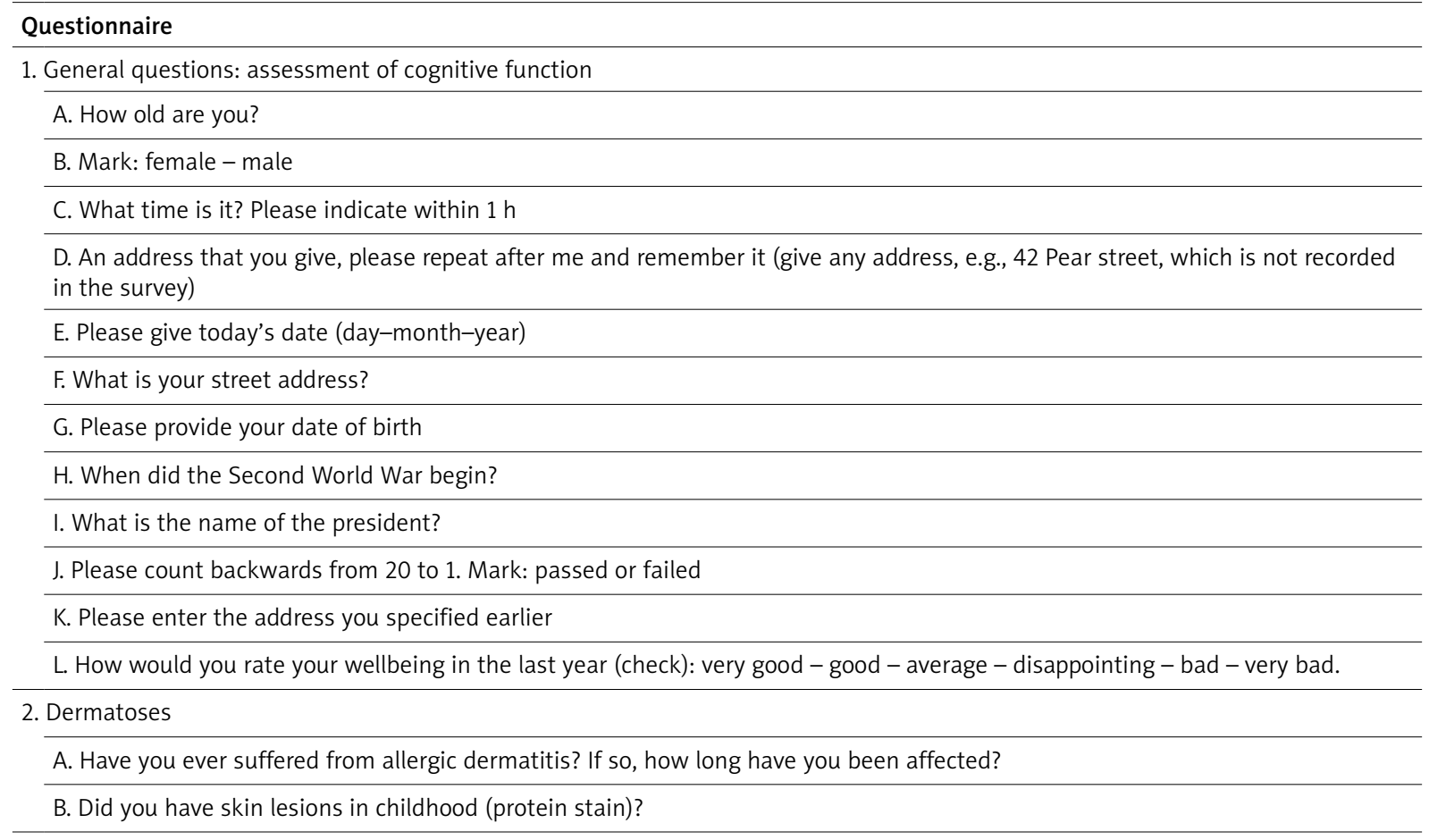


Table 1. Cont.

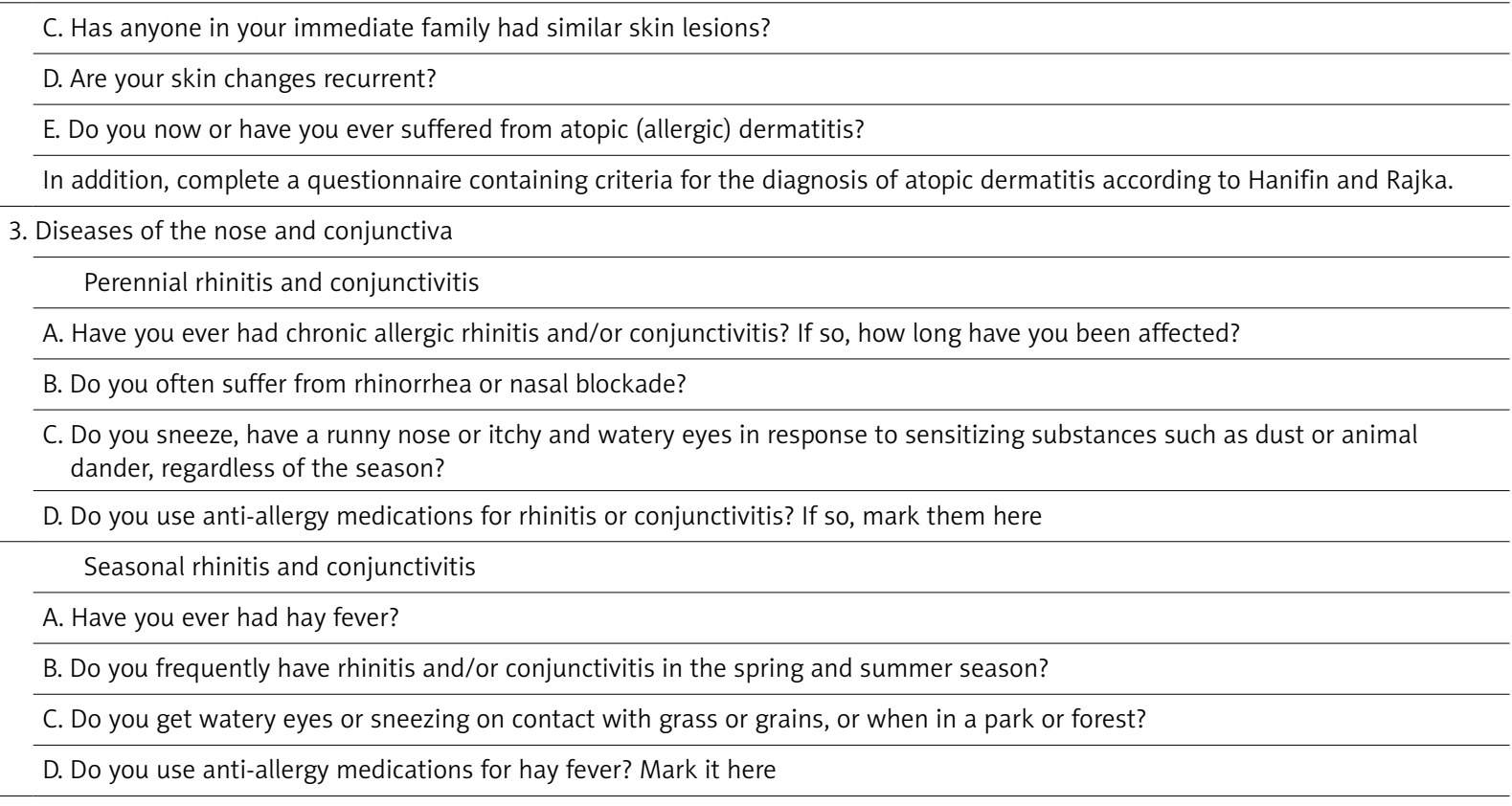

\section{Asthma}

A. Do you ever suffer from asthma? If so, how many years have you had asthma? When were you ill (check)? In childhood - in youth - the age - adults

B. During the past 12 months, did you experience wheezing in the chest or cough?

B1. If so, was it related to rhinitis?

B2. If so, have you had cough or wheezing without colds?

C. Were you ever awakened by a feeling of tightness or compression in the chest during the last 12 months?

D. Were you ever awakened by an attack of shortness of breath in the last 12 months?

E. Did you ever wake up coughing in the last 12 months?

F. Did you have an asthma attack in the last 12 months?

G. Have you ever used asthma medications?

5. Do you smoke?

6. Do you suffer from the following diseases:

A. Heart disease?

B. Diseases of the gastrointestinal tract?

The key to calculate the score from the survey: The questionnaire has 6 modules. Each module has an individual score as described below.

Part One. Quick test of mental ability: for each correct answer: 1 point. A normal state should be $>6$ points, 4-6 points indicates moderate impairment, and 0-3 points indicates severe impairment. In the case of a result of less than 4 points, the patient does not complete the rest of the survey. In other cases, the person accompanying the patient is allowed to help the patient.

Part Two. Diagnosis of perennial rhinitis and conjunctivitis. For a positive answer to each question: 1 point. A minimum of two points confirms the diagnosis.

Seasonal rhinitis and conjunctivitis:

For a positive answer to each question: 1 point. A minimum of two points confirms the diagnosis.

Part Three. Diagnosis of asthma:

An answer of "yes" to Question A or "no" to Question A, but affirmative answers to at least three other questions confirms the diagnosis.

Part Four. Diagnosis of skin allergic diseases

An answer of "yes" to Question A or "no" to Question A, but affirmative answers to all of the other questions confirms the diagnosis.

Parts Five and Six contain descriptive questions only with positive or negative questions. 


\section{Skin prick test (SPT)}

Skin prick tests were performed according to the Polish Society of Allergology [11]. First, the $10 \mathrm{mg} / \mathrm{ml}$ histamine positive control was placed, followed by the negative control, which consisted of the diluent for the other allergens (Allergopharma, Germany). A set of allergens was then applied to the skin in quantities of an approximately $0.05 \mathrm{ml}$ drop in the following order: Dermatophagoides pteronyssinus, Dermatophagoides farinae, cat, dog, grass/wheat, rye, hazel, alder, birch, Artemisia, plantain, Alternaria, and Cladosporium (50 $000 \mathrm{BE} / \mathrm{ml}$ ). The histamine positive control response was assessed after $10 \mathrm{~min}$, and the allergen tests were assessed after $20 \mathrm{~min}$. The test result was considered reliable if the wheal reaction to histamine had a diameter of at least $3 \mathrm{~mm}$ with associated erythema of at least $5 \mathrm{~mm}$ in diameter. Instances of skin bubbles larger than the negative control and with a diameter $>3 \mathrm{~mm}$ were considered positive. The presence of a wheal larger than the negative control and with a diameter greater than $3 \mathrm{~mm}$ was considered positive.

Tests were performed after the discontinuation of antihistamine drugs for at least 1 week. The patients had no symptoms of acute infection within 4 weeks and did not use antidepressants or prednisolone $>10 \mathrm{mg}$ per day. Topical corticosteroid and anesthetic preparations were not used.

\section{Serum concentration of total IgE and specific lgE (slgE)}

Ten milliliters of blood from each subject was collected in citrate and then centrifuged to yield serum for further studies. Samples were stored at 2 to $-8^{\circ} \mathrm{C}$, and IgE assays were performed within one week after blood collection. The determinations were made using the fluoroimmunoenzymatic Pharmacia CAP System FEIA (ThermoFisher, Sweden). The sensitivity of the method determines the concentration to below $2 \mathrm{kU} / \mathrm{l}$. The concentrations of slgE against allergens including D. pteronyssinus, $D$. farinae, cat dander, dog, grass, grain, alder, hazel, birch, Artemisia, plantain, Alternaria, and Cladosporium, were determined with the fluoroimmunoenzymatic Pharmacia CAP System FEIA (ThermoFisher, Sweden). Values above $0.35 \mathrm{kU} / \mathrm{I}$ were considered positive [10]. The resulting concentrations of total IgE and slgE units are presented in $\mathrm{kU} / \mathrm{l}$ as the geometric mean with one standard deviation.

\section{Statistical analysis}

The rates of allergic diseases are presented in the form of prevalence associated with gender and age. Prevalence is presented as the average percentages of all study centers with 95\% confidence intervals. Odds ratios for allergy suspicion based on the selected parameters were calculated, and the data are presented with

Table 2. Characteristics of the study population

\begin{tabular}{|c|c|c|c|}
\hline Characteristics & $\begin{array}{l}\text { With probable allergy } \\
\qquad N=234\end{array}$ & $\begin{array}{l}\text { Without probable allergy } \\
\qquad N=826\end{array}$ & $P$-value ${ }^{\wedge}$ \\
\hline Age (mean \pm SD) [years] & $68.1 \pm 6.2$ & $69.1 \pm 8.4$ & $\mathrm{NS}^{*}$ \\
\hline Female, $n(\%)$ & $127(54.3)$ & $463(56.1)$ & NS \\
\hline \multicolumn{4}{|c|}{ Alzheimer's disease status, $n$ (\%): } \\
\hline $1^{\circ}$ & $59(25.2)$ & $164(19.9)$ & 0.03 \\
\hline $11^{\circ}$ & $110(47.0)$ & $434(52.5)$ & 0.04 \\
\hline$I I I^{\circ}$ & $60(25.6)$ & $225(27.2)$ & NS \\
\hline $\mathrm{IV}^{\circ}$ & $5(2.1)$ & $57(6.9)$ & 0.03 \\
\hline \multicolumn{4}{|l|}{ Education level, $n(\%)$ : } \\
\hline None & $35(15.0)$ & $220(26.6)$ & 0.03 \\
\hline Basic & $52(22.2)$ & $284(34.4)$ & 0.02 \\
\hline Average & $55(23.5)$ & $212(25.7)$ & NS \\
\hline High & $92(39.3)$ & $110(13.3)$ & 0.002 \\
\hline \multicolumn{4}{|l|}{ Family history, $n(\%)$ : } \\
\hline Bronchial asthma & $34(14.5)$ & $18(2.2)$ & 0.003 \\
\hline Allergic dermatoses & $45(19.3)$ & $31(3.8)$ & 0.002 \\
\hline Rhinitis & $51(21.8)$ & $195(23.6)$ & NS \\
\hline Children of respondents & $157(67.0)$ & $294(35.6)$ & 0.01 \\
\hline
\end{tabular}

${ }^{\wedge} \chi^{2}$ test with Bonferroni correction, *Student's unpaired t-test. 
95\% confidence intervals. The results of skin prick tests (SPT) and allergen-specific IgE (slgE) are presented as frequencies averaged from all 16 centers with $95 \%$ confidence intervals and standardization for age and sex. To compare the SPT results and IgE levels, we used the $\chi^{2}$ test. IgE values are presented as geometric means with standard deviations. After logarithmic transformation of the IgE results, the differences between groups were compared using Student's t-test. Multiple linear regression analysis was used to explore the relationships between total IgE and selected characteristics of the group. The statistical analyses included Bonferroni correction. $P$-value $<0.05$ was considered significant.

\section{Results}

The analysis included 1060 subjects with a mean age of $69.2 \pm 5.1$ years (range: 60-97 years; 590 women and 470 men). Suspicion of atopy was diagnosed in 234 (22.1\%) patients, including 127 women (21.5\% of women) and 107 men (22.8\% of men). The characteristics of the group are shown in Table 2 . In the selected subgroups of patients with atopic diseases, the group of women was significantly larger than the group of men $(p<0.05)$. The average morbidity associated with age and sex in this population of people with AD and bronchial asthma (BA) was $2.9 \%$ (95\% Cl: 2.1-3.4), for atopic dermatitis was 0.6\% (95\% Cl: 0.5-2.2), for perennial allergic rhinitis (PAR) was $11.1 \%$ (95\% Cl: 9.9-15.4), for seasonal allergic rhinitis (SAR) was 6.6\% (95\% Cl: 4.8-8.6) and for polymorphous atopic disease was 4.4\% (95\% Cl: 3.1-5.3). The mean duration of allergic disease was $18.8 \pm 11.4$ years. Suspected atopic disease prevailed in the cities compared to rural areas $(p<0.05)$.

\section{Skin prick tests for aeroallergens in the subgroup of atopy}

In 182 (77.8\%) patients, at least one positive skin test to allergens was observed.

The results of the skin tests are shown in Table 2. The most frequent positive results were recorded for the following allergens: mixed grass/grain $-33.2 \%$, D. pteronyssinus $-25.6 \%$ and Alternaria $-20.4 \%$. Allergy associated with 2, 3 or more allergens was present in 121 (51.7\%) patients. In a subgroup without allergy, 25 (3.2\%) patients had one or more positive tests however without clinical symptoms.

\section{Concentration of total and allergen-specific IgE}

Patients with a positive questionnaire for allergic disease had statistically higher values of IgE (145.8 $\pm 62.3 \mathrm{kU} / \mathrm{l})$ in relation to other subjects $(45.2 \pm 29.5 \mathrm{kU} / \mathrm{l})$ $(p<0.05)$. There were no significant differences in IgE and AD severity. The concentrations of allergen-specific IgEs are shown in Table 3. As with the results of the skin tests, the most frequent IgE-positive results were recorded for grass (29.1\%), D. pteronyssinus (30.1\%) and Alternaria (19.3\%). In all analyzed patients with AD, the possibility of allergic disease occurred with the odds ratio 0.67 (95\% Cl: 0.55-0.73).

\section{Discussion}

The phenomenon of increasing allergy in elderly patients is new in the XXI century [12-15]. There are more and more studies of this problem especially regarding bronchial asthma. The incidence of asthma in the elderly ranged from $4 \%$ to $6 \%$, but with significant underestimation by the authors. The problem of underdiagnosis and undertreatment of asthma in the elderly population is unquestionable and it is subject of several studies [16, 17]. A similar problem was observed also in patients with AD. Appropriate treatment of asthma leads to a cognitive improvement also in patients with mild and moderate $\operatorname{AD}[18,19]$. However there was no information about prevalence other allergic diseases in patients with cognitive impairment. Is it a problem in analyzed patients? The positive answer follows from the obtained results. The prevalence of allergic rhinitis, atopic dermatitis is significant but lower in comparison with results in healthy elderly subjects in other studies $[13,20,21]$. This differences is at a level of $5-10 \%$. These findings are very interesting and could provide that immunomodulation in $A D$ may function partially protective to allergy. This may be due to the balance shift in the direction of lymphocytes Th1. Typical of the elderly but also of the allergy, Th2 dominance would change in this type of patients. However, this requires precise confirmation in larger numbers of patients.

Table 3. Incidence of positive SPT and specific IgE determinations

\begin{tabular}{lcc}
\hline \multirow{2}{*}{ Allergen } & \multicolumn{2}{c}{ Percentage of positive results (95\% Cl) } \\
\cline { 2 - 3 } & SPT & slgE \\
\hline D. pteronyssinus & $25.6(22.4-27.8)$ & $30.1(28.4-33.7)$ \\
\hline D. farinae & $18.8(16.8-20.4)$ & $25.8(20.5-27.4)$ \\
\hline Cat & $10.3(9.5-12.7)$ & $13.7(11.5-16.9)$ \\
\hline Dog & $6.4(5.0-7.8)$ & $15.8(13.2-17.5)$ \\
\hline Grass & $33.2(31.2-36.7)$ & $29.1(26.8-32.7)$ \\
\hline Rye & $12.8(10.2-14.5)$ & $14.6(11.5-15.1)$ \\
\hline Hazel & $9.4(5.6-11.7)$ & $9.1(5.6-10.1)$ \\
\hline Alder & $5.9(7.8-9.4)$ & $5.5(3.2-6.8)$ \\
\hline Birch & $13.9(12.1-15.5)$ & $16.2(13.2-17.5)$ \\
\hline Artemisia & $11.4(8.5-12.6)$ & $13.2(11.3-15.3)$ \\
\hline Plantain & $5.8(3.1-10.7)$ & $5.7(4.1-7.4)$ \\
\hline Alternaria & $19.2(17.4-22.2)$ & $19.3(18.2-22.7)$ \\
\hline Cladosporium & $10.4(8.4-12.6)$ & $10.7(8.7-13.6)$ \\
\hline
\end{tabular}


Besides $A D$, analyzed patients with allergy confirmation had better education and more often had a positive family history of allergic diseases.

The presence of positive tests to common inhalant allergens especially to house dust mites and grass pollen are similar as in other elderly people with allergy. Additionally, similar compatibility concerns the concentration of total and specific lgE [13, 21, 22]

This leads to a conclusion that allergic diseases in patients with $A D$ are present as in other elderly subjects. However there is a frequently significant problem with obtaining medical history and performing allergy diagnostic procedures. Therefore, it seems that allergy in patients with $A D$ is often underdiagnosed. The limitation of this study is lack of analyzed patients with the most severe, fifth degree of $A D$. However, in this form of the disease, allergy has no impact on quality of life in such subjects. Another limitation of the study is the lack of assessment of quality of life in examined patients. It is a very difficult task in patients with cognitive impairment because all questionnaires of quality of life with a real influence of allergic disease are inadequate and too complicated for them. However similar assessment in elderly patients with allergy but without cognitive impairment indicates a significant deterioration in the quality of life. The treatment of allergic disease could improve not only quality of life but improve the mental function as it was demonstrated in bronchial asthma.

\section{Conclusions}

The prevalence of allergic diseases in patients with Alzheimer's diagnosis is significant but lower as compared to other elderly people. This problem may require more medical attention.

\section{Conflict of interest}

The authors declare no conflict of interest.

\section{References}

1. Bodtger U, Poulsen LK, Linneberg A. Rhinitis symptoms and IgE sensitization as risk factors for development of later allergic rhinitis in adults. Allergy 2006; 61: 712-6.

2. Busse PJ. Allergic respiratory disease in the elderly. Am J Med 2007; 120: 498-502.

3. Weiner MW, Aisen PS, Jack CR, et al. The Alzheimer's disease neuroimaging initiative: progress report and future plans. Alzheimers Dement 2010; 6: 202-11.

4. Alzheimer's Association: 2009. Alzheimer's disease facts and figures. Alzheimers Dement 2009; 5: 234-70.

5. Cukierman-Yaffe T, Gerstein HC, Williamson JD, et al. Relationship between baseline glycemic control and cognitive function in individuals with type 2 diabetes and other cardiovascular risk factors: the action to control cardiovascular risk in diabetes-memory in diabetes (ACCORD-MIND) trial. Diabetes Care 2009; 32: 221-6.
6. Morrison JR. Dementia, delirium, and amnestic and other cognitive disorders. In: DSM-IV made easy: the clinician's guide to diagnosis. Morrison JR (ed.). Guilford Press, New York 1995; 11-53.

7. World Health Organization. The ICD-10 classification of mental and behavioural disorders: clinical descriptions and diagnostic guidelines. World Health Organization, Geneva.

8. Folstein MF, Folstein SE, McHugh PR. "Mini-mental state". A practical method for grading the cognitive state of patients for the clinician. J Psychiatr Res 1975; 12: 189-98.

9. Respiratory Health Survey II. ECRHS II Screening questionnaire. Project Leaders: Burney P, Jarvis D. 2007, www.ecrhs.org.

10. Pharmacia CAP System FEIA - manual brochure 2002.

11. Komisja ds. Standardów Zarządu Głównego Polskiego Towarzystwa Alergologicznego pod red. J. Kruszewskiego. Standardy w alergologii. Cz. I, Dom Wydawniczy Benkowski 2003; 9-29.

12. Ariano R, Panzani RC. Late onset asthma in the elderly and its relationship with atopy. Eur Ann Allergy Clin Immunol 2012; 44: 35-41.

13. Bozek A, Jarzab J. Epidemiology of IgE dependent allergic diseases in elderly patients with Poland. Am J Rhinol Allergy 2013; 27: 140-3.

14. Epstein TG, Ryan PH, LeMasters GK, et al. Poor asthma control and exposure to traffic pollutans and obesity in older adults. Ann Allergy Asthma Immunol 2012; 108: 423-8.

15. Huss K, Naumann PL, Mason PJ, et al. Asthma severity, atopic status, allergen exposure and quality of life in elderly persons. Ann Allergy Asthma Immunol 2001; 86: 524-30.

16. van Schayck CP, van Der Heijden FM, van Den Boom G, et al. Underdiagnosis of asthma, is the doctor or the patient to blame? The DIMCA project. Thorax 2000; 55: 562-5.

17. Banerjee DK, Lee GS, Malik SK, Daly S. Underdiagnosis of asthma in the elderly. Br J Dis Chest 1987; 81: 23-9.

18. Eriksson UK, Gatz M, Dickman PW, et al. Asthma, eczema, rhinitis and the risk for dementia. Dement Geriatr Cogn Disord 2008; 25: 148-56.

19. Bozek A, Jarzab J. Improved activity and mental function related to proper antiasthmatic treatment in elderly patients with Alzheimer's disease. Allergy Asthma Proceedings 2011; 32: 342-5.

20. Wolkewitz M, Rothenbacher D, Low M, et al. Liftime prevalence of self-reported atopic diseases in a population-based sample of elderly subjects: results of the ESTHER study. $\mathrm{Br}$ J Dermatol 2007; 156: 693-7.

21. Becerril Angeles M, Vazquez Merino CL, Angeles Garay U, et al. Prevalence of allergic diseases in the elderly. Rev Alerg Mex 2008; 55: 85-91.

22. Asero R, Conte M, Senna GE. Features of sensitization to airborne allergens among extra-European immigrants living in 2 distinct areas of Nothern Italy. Eur Ann Allergy Clin Immunol 2012, 44: 107-12. 